\title{
Dietary n-3 PUFAs Deficiency Increases Vulnerability to Inflammation-Induced Spatial Memory Impairment
}

\author{
Jean-Christophe Delpech ${ }^{1,2}$, Aurore Thomazeau' ${ }^{1,2}$, Charlotte Madore ${ }^{1,2}$, Clementine Bosch-Bouju ${ }^{1,2}$, \\ Thomas Larrieu $^{1,2}$, Chloe Lacabanne ${ }^{1,2}$, Julie Remus-Borel ${ }^{1,2}$, Agnès Aubert ${ }^{1,2}$, Corinne Joffre ${ }^{1,2}$, \\ Agnès Nadjar ${ }^{*, 1,2,3}$ and Sophie Layé*,1,2,3 \\ 'INRA, Nutrition et Neurobiologie Intégrée, UMR 1286, Bordeaux, France; '2University of Bordeaux, Nutrition et Neurobiologie Intégrée, \\ UMR 1286, Bordeaux, France
}

\begin{abstract}
Dietary n-3 polyunsaturated fatty acids (PUFAs) are critical components of inflammatory response and memory impairment. However, the mechanisms underlying the sensitizing effects of low n-3 PUFAs in the brain for the development of memory impairment following inflammation are still poorly understood. In this study, we examined how a 2-month n-3 PUFAs deficiency from pre-puberty to adulthood could increase vulnerability to the effect of inflammatory event on spatial memory in mice. Mice were given diets balanced or deficient in n-3 PUFAs for a 2-month period starting at post-natal day 21, followed by a peripheral administration of lipopolysaccharide (LPS), a bacterial endotoxin, at adulthood. We first showed that spatial memory performance was altered after LPS challenge only in $\mathrm{n}-3$ PUFA-deficient mice that displayed lower n-3/n-6 PUFA ratio in the hippocampus. Importantly, long-term depression (LTD), but not longterm potentiation (LTP) was impaired in the hippocampus of LPS-treated n-3 PUFA-deficient mice. Proinflammatory cytokine levels were increased in the plasma of both n-3 PUFA-deficient and n-3 PUFA-balanced mice. However, only n-3 PUFA-balanced mice showed an increase in cytokine expression in the hippocampus in response to LPS. In addition, n-3 PUFA-deficient mice displayed higher glucocorticoid levels in response to LPS as compared with n-3 PUFA-balanced mice. These results indicate a role for n-3 PUFA imbalance in the sensitization of the hippocampal synaptic plasticity to inflammatory stimuli, which is likely to contribute to spatial memory impairment. Neuropsychopharmacology (20I5) 40, 2774-2787; doi: 10.1038/npp.2015. I27; published online 27 May 2015
\end{abstract}

\section{INTRODUCTION}

Lipids are building blocks of the brain (Bazinet and Laye, 2014), a structure which is highly enriched in polyunsaturated fatty acids (PUFAs) from the n-3 and n- 6 families. The brain contains particularly high amounts of longchain PUFAs, docosahexaenoic acid (DHA, 22:6n-3) and arachidonic acid (AA, 20:4 n-6). Mammals cannot synthesize de novo their precursors, namely $\alpha$-linolenic acid (18:3 $\mathrm{n}-3$ ) and linoleic acid (LA, 18:2 n-6), respectively, so they have to be provided through dietary means. Adequate precursor supplies are necessary to maintain sufficient brain levels of the long-chain PUFAs, DHA and AA that are crucial for the normal function of the brain (Bazinet and Laye, 2014). Clinical and epidemiological studies consistently reported that low levels of dietary or blood content of long-chain n-3 PUFAs are inversely correlated to cognitive performance (recently reviewed in (Luchtman and Song, 2013; Joffre et al, 2014). Although in various animal models,

* Correspondence: Dr A Nadjar or Dr S Layé, Nutrition et Neurobiologie Intégrée, University of Bordeaux, I46 rue Leo Saignat, Bordeaux 33076, France, Tel: +00 3355757 1085, Fax: +33 55757 I227, E-mail: agnes.nadjar@u-bordeaux.fr or sophie.laye@bordeaux.inra.fr

${ }^{3}$ These authors contributed equally to this work.

Received 3 February 2015; revised 7 April 2015; accepted 24 April 2015; accepted article preview online 7 May 2015 the reduction of brain DHA levels through nutritional n-3 PUFA deficiency has been associated with memory deficits and neuronal plasticity alteration (Fedorova and Salem, 2006; Arsenault et al, 2011; Lafourcade et al, 2011; Labrousse et al, 2012; Moranis et al, 2012; Luchtman and Song, 2013; Joffre et al, 2014), the mechanisms underlying these effects are still poorly understood.

In addition to their effects on neuronal plasticity, PUFAs are neuroimmunomodulators and regulate the activity of brain innate immune cells, so-called 'microglia' (Orr and Bazinet, 2008; Bazan et al, 2011; Lalancette-Hebert et al, 2011; Calder, 2013; Chang et al, 2015). A simplistic view is that n-3 PUFAs form the basis of lipid derivatives with anti-inflammatory properties, whereas n- 6 PUFAs are the precursors of proinflammatory mediators, and stimulate the production and activity of proinflammatory factors such as cytokines (Farooqui et al, 2007; Orr et al, 2013; Serhan et al, 2014). As a result, we have previously demonstrated that low dietary intake of $n-3$ PUFAs promotes neuroinflammatory responses through the regulation of microglial cells activity and polarization toward a pro-inflammatory phenotype, whereas n-3 PUFA dietary supplementation is rather antiinflammatory (De Smedt-Peyrusse et al, 2008; Mingam et al, 2008; Labrousse et al, 2012; Madore et al, 2014; Delpech et al, 2015a). A dramatic reduction in the dietary supply of $n-3$ PUFAs could thus contribute to the sensitization of the brain 
immune response to further inflammation, and thus to the development of spatial memory disorders.

Neuroinflammation is a common early feature of most peripheral and central diseases and is known to alter cognition, in particular spatial memory (for review, (Cunningham and Sanderson, 2008; Yirmiya and Goshen, 2011). Neuroinflammation is characterized by the brain synthesis and release of pro-inflammatory mediators known to control neuronal function (Hanisch and Kettenmann, 2007; Cunningham and Sanderson, 2008; Yirmiya and Goshen, 2011; Pascual et al, 2012; Delpech et al, 2015b). Pro-inflammatory factors including interleukin-1 beta (IL-1 $\beta$ ), interleukin-6 (IL-6), and tumor necrosis factor alpha (TNF- $\alpha$ ) have been directly linked to impaired neuronal plasticity in various animal models (Yirmiya and Goshen, 2011; Delpech et al, 2015b). Several groups now suggest an inverted U-shape relationship between the hippocampal inflammatory state and cognitive functions, whereby a small increase in the inflammatory state is beneficial, whereas a larger increase is detrimental to behavioral and neuronal plasticity (Goshen et al, 2007; Goshen et al, 2009; Yirmiya and Goshen, 2011; Reshef et al, 2014). This hypothesis was recently corroborated by an elegant study demonstrating that depletion in microglia (the brain producer of pro-inflammatory cytokines) induces learning impairments in multiple learning and memory tasks (Parkhurst et al, 2013).

Knowing the role of n-3 PUFAs in synaptic plasticity and neuroinflammatory responses, we hypothesized that low n-3 PUFAs is a vulnerability factor for the development of memory impairment in inflammatory situation. Our objective was then to identify the mechanisms underlying the vulnerability associated with n-3 PUFA deficiency to memory impairment in response to an immune challenge. For this purpose, mice were fed with n-3 PUFA deficient $v s$ n-3 PUFA-balanced diet for 2 months, starting at weaning. Adult mice were then injected peripherally with lipopolysaccharide (LPS), a potent producer of brain cytokines (Laye et al, 1994). We assessed spatial memory and hippocampal neuronal plasticity, blood and brain cytokine production as they are known to modulate neuronal functions, as well as glucocorticoid levels, a potent immunosuppressant (Coutinho and Chapman, 2011). Our results showed that a LPS challenge altered spatial memory performance only in n-3 PUFA-deficient mice and this was paralleled by impairment of long-term synaptic plasticity, namely longterm depression (LTD), in these animals. We also found that the brain inflammatory response to LPS challenge was reduced in n-3 PUFA-deficient animals compared with n-3 PUFA-balanced mice while blood concentrations of inflammatory factors were similar in both groups. Finally, glucocorticoid levels were significantly higher in the blood of n-3 PUFA-deficient mice after LPS treatment.

\section{MATERIALS AND METHODS}

\section{Animals}

All experiments were performed according to criteria of the European Communities Council Directive (50120103-A). Behavioral, electrophysiological, and biochemical experiments were performed on $\mathrm{C} 57 \mathrm{Bl6} / \mathrm{J}$ male mice obtained from Charles River (Arbresle, France). Mice were maintained under standard housing conditions on corncob litter in a temperature $\left(23 \pm 1^{\circ} \mathrm{C}\right)$ - and humidity (40\%)-controlled animal room with a 12-h light/dark cycle $(0700-1900 \mathrm{~h})$, with ad libitum access to food and water. All tests were conducted during the light period.

\section{Diets}

Dams were kept on a standard diet (A04, 3.1\% lipids, SAFE, Augy, France) until the age of 3 weeks. At post-natal day 21, pups were fed with a diet containing $5 \%$ of an lipid mixture rich in rapeseed oil (rich in $\alpha$-linolenic acid, 18:3n-3; ' $n-3$ PUFA-balanced diet') or $5 \%$ fat in the form of sunflower oil (rich in LA, 18:2n-6; 'n-3 PUFA-deficient diet') for 2 months until the end of the experiments (Mingam et al, 2008; Lafourcade et al, 2011; Moranis et al, 2012). Pellets were prepared by UPAE-INRA (Jouy-en-Josas, France) and stored at $4{ }^{\circ} \mathrm{C}$. Fatty acid composition was regularly controlled via gas chromatography analyses of organic extracts from manufactured food pellets as previously described (Lafourcade et al, 2011; Larrieu et al, 2014; Madore et al, 2014).

\section{Treatments}

To induce an inflammatory reaction, a dose of $125 \mu \mathrm{g} / \mathrm{kg}$ of LPS (Escherichia coli, 0127:B8, Sigma-Aldrich, Lyon, France), diluted in saline $(\mathrm{NaCl} 0.9 \%)$ was intraperitoneally injected between 9 and 11am. LPS is a component of the cell wall of Gram-negative bacteria and represents a useful model for investigating changes that accompany brain inflammation such as spatial memory impairment (Laye et al, 1994; Mingam et al, 2008; Delpech et al, 2015a). Control mice received an injection of saline solution (0.9\%). Food consumption (assessed by weighing the pellets) and body weight were monitored $24 \mathrm{~h}$ after LPS injection as a marker of sickness (Laye et al, 2000). For qPCR experiments, Bioplex assays, and corticosterone measurements, mice were quickly anesthetized by isoflurane inhalation and killed by decapitation $2,6,12$, or $24 \mathrm{~h}$ after treatment. For lipid measurements, electrophysiological studies, and spine counting, mice were quickly anesthetized by isoflurane and killed by decapitation $24 \mathrm{~h}$ after treatment. Twenty-four hours after the treatment, mice have recovered from the sickness symptoms and present a normal locomotor activity, allowing us to investigate their spatial memory abilities (Delpech et al, 2015a).

\section{Analysis of Fatty Acid Contents}

Fatty acids from the hippocampus were analyzed as previously described (Labrousse et al, 2012; Madore et al, 2014; Delpech et al, 2015a). Fatty acid composition is expressed as the percentage of total fatty acids.

\section{Plasma Cytokine Assay}

Cytokine assays were performed as previously described (Moranis et al, 2012). The limit of detection was $1.1 \mathrm{pg} / \mathrm{ml}$ for IL-6, $2.3 \mathrm{pg} / \mathrm{ml}$ for TNF- $\alpha, 5.4 \mathrm{pg} / \mathrm{ml}$ for IL- $1 \beta$, and $2 \mathrm{pg} / \mathrm{ml}$ for IL-10. Briefly, samples diluted $1 / 2$ were added to 
a 96 -well microtiter plate $(25 \mu \mathrm{l} /$ well $)$ coated with beads (Millipore, France), covered with aluminum foil and incubated overnight on a shaker at $4{ }^{\circ} \mathrm{C}$ in the dark. After removal of sample using a magnet, beads were incubated with detection antibodies for $1 \mathrm{~h}$ at room temperature while shaking, followed by streptavidin-PE for $30 \mathrm{~min}$. The beads were then resuspended in $150 \mu \mathrm{l}$ Sheath Fluid and analyzed using the BioPlex 200 system (Bio-Rad, France). The reader was set to read a minimum of 50 beads with an identical fluorescence expressed as the median fluorescence intensity. Median fluorescence intensity readings were converted to $\mathrm{pg} / \mathrm{ml}$ using calibration curves prepared with cytokine standards included in the kit. Results were expressed in $\mathrm{pg} / \mathrm{ml}$.

\section{Quantitative Real-Time PCR}

Total RNA was extracted from whole hippocampi using TRIzol (Invitrogen, Life Technologies ${ }^{\mathrm{TM}}$ ). RNA purity and concentration were determined using a Nanodrop spectrophotometer (Nanodrop technologies, Wilmington, DE). One microgram of RNA was reverse-transcribed to synthesize cDNA using Superscript III (Invitrogen, Life Technologies $^{\mathrm{TM}}$ ) and random hexamers according to the manufacturer's protocol (Labrousse et al, 2012; Madore et al, 2013; Madore et al, 2014; Delpech et al, 2015a). Quantitative PCR was performed to measure cytokine expression using the Applied Biosystems (California, USA) assay-on-demand gene expression protocol as previously described (Mingam et al, 2008; Delpech et al, 2015a). In brief, cDNA was amplified by real-time PCR where a target $\mathrm{cDNA}$ and a reference cDNA ( $\beta 2$-microglobulin) were amplified simultaneously using an oligonucleotide probe with a $5^{\prime}$ fluorescent reporter dye (6-FAM) and a $3^{\prime}$ quencher dye (NFQ). Fluorescence was determined on an ABI PRISM 7500sequence detection system (Applied Biosystems, California, USA). Data were analyzed using the comparative threshold cycle (Ct) method, results are expressed as relative fold change (Mingam et al, 2008; Madore et al, 2013; Madore et al, 2014; Delpech et al, 2015a) to control target mRNA expression.

\section{Plasmatic Corticosterone Analysis}

Trunk blood collection in ethylenediaminetetraacetic acidlined tubes was performed during diurnal rise period, previously determined to occur $60 \mathrm{~min}$ before lights off. Corticosterone was measured with an in-house radio immunoassay in the plasma as previously described (Richard et al, 2010; Larrieu et al, 2014). Briefly, after steroid extraction with absolute ethanol, total corticosterone was measured by competition between cold corticosterone (B) and $3 \mathrm{H}-\mathrm{B}\left(\mathrm{B}^{*}\right)$ by a specific anticorticosterone antibody provided by Dr H. Vaudry (University of Rouen, France).

\section{Spine Counting}

Morphological analyses were conducted $24 \mathrm{~h}$ after LPS or saline treatment. Brains were quickly removed, washed in phosphate-buffered saline and processed for staining of individual neurons following the manufacturer's instructions for the rapid Golgi kit (FD Neurotech, Columbia, MD, USA). Golgi-stained brain slices of $100 \mu \mathrm{m}$ containing the dorsal hippocampus were used for morphological analysis. Images were acquired by a trained experimenter blind to the conditions using motorized Leica DM5000 microscope at a $\times 63$ magnification. The images were acquired using a CCD Coolsnap camera and Metamorph software.

For analysis, we randomly selected pyramidal neurons from the CA1 region of the dorsal hippocampus that were fully penetrated by the Golgi coloration and clearly distinguishable from other neurons. Neuron reconstruction was performed using the Fiji software (Schindelin et al, 2012). Between three and five neurons were selected from each animal. Spine density in these neurons was determined by counting the number of spines on at least three basal and three apical dendritic segments of $10 \mu \mathrm{m}$ in length. Segments from dendrites situated as far from the cell body as possible, with no overlap with other dendrites were randomly selected. Primary dendrites were never used for analysis as their thickness hampers detection of spines. Spine density was calculated per $10 \mu \mathrm{m}$ and averaged across the different segments in the same neuron.

\section{Electrophysiological Recordings}

Coronal hippocampal slices $(350 \mu \mathrm{m})$ were prepared from adult mice fed with n-3 PUFA-balanced diet or n-3 PUFAdeficient diet, $24 \mathrm{~h}$ after saline or LPS injection. Slices were prepared with a vibrating blade microtome (VT1000S, Leica Microsystems, Nussloch, Germany) in an oxygenated artificial cerebrospinal fluid (ACSF) containing (in mM): 23 $\mathrm{NaHCO}_{3}, 87 \mathrm{NaCl}$, 75 Sucrose, 25 Glucose, $4 \mathrm{MgCl}_{2}, 2.5 \mathrm{KCl}$, $0.5 \mathrm{CaCl}_{2}, 1.25 \mathrm{NaH}_{2} \mathrm{PO}_{4}$. Slices were stored at room temperature and recordings were started after at least $1 \mathrm{~h}$ of rest. ACSF solution for storage contained (in $\mathrm{mM}$ ): 23 $\mathrm{NaHCO}_{3}, 130 \mathrm{NaCl}, 11$ Glucose, $2.4 \mathrm{MgCl}_{2}, 2.5 \mathrm{KCl}, 1.2$ $\mathrm{CaCl}_{2}, 1.2 \mathrm{NaH}_{2} \mathrm{PO}_{4}$. ACSF solution for recordings contained (in nM): $23 \mathrm{NaHCO}_{3}, 130 \mathrm{NaCl}$, 11 Glucose, $1.2 \mathrm{MgCl}_{2}, 2.5$ $\mathrm{KCl}, 2.4 \mathrm{CaCl}_{2}, 1.2 \mathrm{NaH}_{2} \mathrm{PO}_{4}$. Extracellular field excitatory post-synaptic potentials (fEPSPs) were evoked by stimulation $(150 \mu \mathrm{s}, 0.1 \mathrm{~Hz})$ of the Schaeffer collateral pathway afferents (bipolar concentric electrode, Phymep, Paris, France and stimulator A365, WPI, Sarasota FL, USA) and recorded in the CA1 stratum radiatum with borosilicate glass pipettes of 5-10 M $\Omega$ resistance filled with recording ACSF. Recordings were performed at $28^{\circ} \mathrm{C}$ using a temperature control system (TC-344B, Warner Instrument Corporation) and slices were continuously superfused at 3-4 $\mathrm{ml} / \mathrm{min}$ with ACSF containing picrotoxin at a final concentration of DMSO of $0.1 \%$ and of picrotoxin of $100 \mu \mathrm{M}$. Signals were amplified using Multiclamp 700B amplifier (Molecular Devices, Sunnyvale, CA, USA) controlled with pClamp 10.3 software via a Digidata 1440 A interface (Molecular Devices). Stable fEPSPs were recorded for $10 \mathrm{~min}$ at $0.1 \mathrm{~Hz}$ with a stimulus intensity of $40-50 \%$ of the maximum fEPSP. Low frequency stimulation (LFS) protocol consists in $1 \mathrm{~Hz}$ stimulation for $15 \mathrm{~min}$ and high frequency stimulation (HFS) consists in three stimulation trains at $100 \mathrm{~Hz}$ for $1 \mathrm{~s}$ with $20 \mathrm{~s}$ interval between the three stimulation trains. After plasticity protocol, fEPSPs were recorded for $45 \mathrm{~min}$ at $0.1 \mathrm{~Hz}$. For each experiment, fEPSP amplitudes were measured $10 \mathrm{~min}$ before plasticity protocol (LFS or HFS) and up to $45 \mathrm{~min}$ after the 
Table I N-6/n-3 PUFA Ratio Increases in the Hippocampus of n-3 PUFA-Deficient Mice

\begin{tabular}{|c|c|c|c|c|c|c|}
\hline & \multicolumn{2}{|c|}{ n-3-deficient diet } & \multicolumn{2}{|c|}{ n-3-balanced diet } & \multicolumn{2}{|c|}{ Statistics } \\
\hline & $\begin{array}{c}\text { Saline } \\
\text { Mean SD }\end{array}$ & $\begin{array}{c}\text { LPS } \\
\text { Mean SD }\end{array}$ & $\begin{array}{c}\text { Saline } \\
\text { Mean SD }\end{array}$ & $\begin{array}{c}\text { LPS } \\
\text { Mean SD }\end{array}$ & diet effect & interaction \\
\hline \multicolumn{7}{|l|}{ Fatty acids } \\
\hline SFA & $42.57 \pm 0.57$ & $42.78 \pm 0.30$ & $43.16 \pm 0.28$ & $42.92 \pm 0.33$ & & \\
\hline $18: 3 n-6$ & $0.01 \pm 0.01$ & $0.02 \pm 0.01$ & $0.02 \pm 0.00$ & $0.02 \pm 0.02$ & & \\
\hline $20: 2 n-6$ & $0.04 \pm 0.01$ & $0.04 \pm 0.01$ & $0.07 \pm 0.00$ & $0.07 \pm 0.01$ & 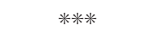 & \\
\hline $20: 3 n-6$ & $0.37 \pm 0.00$ & $0.37 \pm 0.02$ & $0.28 \pm 0.02$ & $0.25 \pm 0.02$ & ***** & \\
\hline $20: 4 n-6$ & $10.46 \pm 0.33$ & $10.17 \pm 0.25$ & $10.96 \pm 0.19$ & $11.06 \pm 0.25$ & ****** & \\
\hline $22: 4 n-6$ & $2.27 \pm 0.15$ & $2.20 \pm 0.04$ & $2.64 \pm 0.05$ & $2.56 \pm 0.07$ & 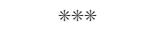 & \\
\hline $22: 5 n-3$ & $0.22 \pm 0.03$ & $0.19 \pm 0.03$ & $0.13 \pm 0.02$ & $0.15 \pm 0.04$ & 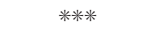 & \\
\hline $22: 6 n-3$ & $15.20 \pm 1.00$ & $14.67 \pm 0.20$ & $12.57 \pm 0.27$ & $|3.3| \pm 0.54$ & 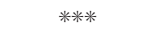 & \\
\hline$n-3$ & $15.50 \pm 0.97$ & $14.94 \pm 0.19$ & $12.78 \pm 0.24$ & $13.54 \pm 0.55$ & 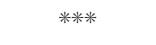 & $*$ \\
\hline$n-6 / n-3$ & $0.90 \pm 0.05$ & $0.90 \pm 0.01$ & $1.28 \pm 0.02$ & $1.23 \pm 0.07$ & **⿻丷木大 & \\
\hline $20: 3 n-9$ & $0.22 \pm 0.03$ & $0.25 \pm 0.03$ & $0.10 \pm 0.01$ & $0.09 \pm 0.01$ & 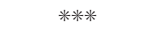 & $*$ \\
\hline PUFA & $29.62 \pm 1.20$ & $28.70 \pm 0.45$ & $29.28 \pm 0.42$ & $30.21 \pm 0.5 I$ & & $*$ \\
\hline
\end{tabular}

Brain fatty acids composition in $\mathrm{n}$-3-balanced diet and $\mathrm{n}$-3-deficient diet mice (\% wt of total fatty acids). Only diet effect and interaction were found positive $($ ***** $p<0.00$ I; *** $<0.01$; * $<0.05)$. No treatment effect was ever observed. $n=4$ per group.

protocol using Clampfit 10.3. All products for ACSF and picrotoxin were provided by Sigma (Saint Quentin Fallavier, France).

\section{Behavioral Test-Y-maze}

Spatial memory was assessed in mice fed with an n-3 PUFAdeficient or -balanced diet, $24 \mathrm{~h}$ after saline or LPS treatment. The Y-maze paradigm was used to assess spatial memory as previously described (Labrousse et al, 2012; Moranis et al, 2012; Delpech et al, 2015a). Mice were handled and weighed every day. All tests were conducted in a sound-attenuated separate experimental room. Behavioral sessions were recorded with a ceiling-mounted video camera and analyzed using specific software (Smart software, Bioseb, France). The apparatus was a Y-shaped maze made of gray plastic. Each arm was $34-\mathrm{cm}$ long, $8-\mathrm{cm}$ wide and $14-\mathrm{cm}$ high. The floor of the maze was covered with used litter from the home cages of all animals and was mixed between sessions to remove olfactory cues. Visual cues were placed in the testing room and kept constant during the whole test. In the first trial of the test, one arm of the Y-maze was closed with a guillotine door and mice were allowed to visit two arms of the Y-maze for $5 \mathrm{~min}$. After 30-min inter-trial interval, mice were placed back in the start arm and allowed free access to the three arms for $5 \mathrm{~min}$. Start and closed arms were randomly assigned for each mouse. Data are presented as the time spent exploring the novel arm during the first 3 min of the second trial.

\section{Y maze (ITI 30 min)}

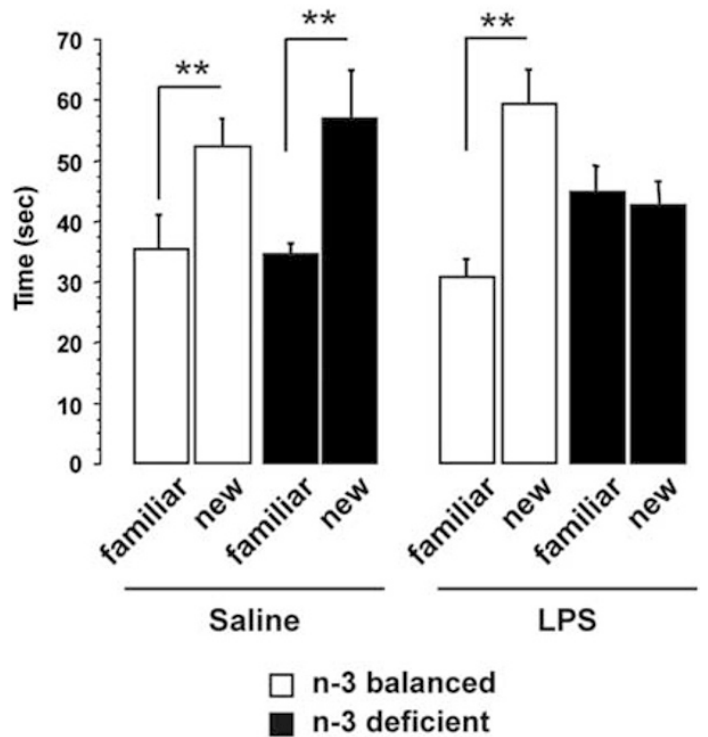

Figure I LPS treatment induces spatial memory impairment in $n-3$ PUFA-deficient mice. Spatial memory was assessed in the Y-maze paradigm for n-3 PUFA-deficient and n-3 PUFA-balanced mice $24 \mathrm{~h}$ after saline or LPS treatment. The graph shows the time spent (in seconds) in the novel or the familiar arm after a 30-min ITI. Only n-3 PUFA-deficient mice injected with LPS exhibited a random exploration of the two arms, as a marker of memory impairment. Data are expressed as mean \pm SEM (*** $<<0.0$ I). N-3 PUFA-deficient/saline: $n=9, n-3$ PUFA-deficient/LPS: $n=10, n-3$ PUFAbalanced/saline $n=10, n-3$ PUFA-balanced/LPS $n=8$. 


\section{Statistical Analyses}

All data are expressed as mean \pm SEM (unless notified 'SD'). For the analysis of PUFA level, spine counting, body weight, and food intake, a two-way ANOVA with diet (n-3-balanced $v s$ n-3-deficient) and treatment (saline $v s$ LPS) as factors was performed. For the analysis of cytokine expression (plasma and brain) and plasma corticosterone concentration, a two-way ANOVA with diet (n-3-balanced $v s \mathrm{n}$-3-deficient) and treatment (saline $v s$ LPS) as factors was performed at each time point. In all cases, when a significant interaction was reported, ANOVAs were followed by post hoc Bonferroni test comparisons. For Y-maze analyses, a three-way ANOVA with treatment and genotype as betweensubjects factors and arm (novel $v s$ familiar) as the withinsubjects factor was performed. Specific comparisons between novel and familiar arms were assessed by paired Student's $t$-tests. Statistical significance was set at $p<0.05$. For electrophysiological recordings, fEPSPs were normalized to $10 \mathrm{~min}$ baseline prior to plasticity induction. Significance of synaptic plasticity was tested for each condition using a paired $t$-test between normalized baseline value and normalized fEPSP value at $45 \mathrm{~min}$ after plasticity induction. To compare plasticity between n-3 PUFA-balanced and n-3 PUFA-deficient animals, we used a Mann-Whitney test. To compare time course of synaptic plasticity for each diet, a two-way ANOVA with time and treatment (saline $v s$ LPS) as factors was performed and when a significant interaction was reported, ANOVAs were followed by post hoc Bonferroni test comparisons.

\section{RESULTS}

\section{N-6/n-3 PUFA Ratio Increases in the Hippocampus of n-3 PUFA-Deficient Mice}

We measured n-3 and n-6 PUFA contents in the hippocampus of mice fed with an n-3-deficient or n-3-balanced diet for 2 months, starting at P21 (Table 1). Manipulating PUFA contents for that period of time was enough to significantly increase $n-6 / n-3$ ratio in $n$-3-deficient animals (two-way ANOVA with diet and treatment as factors; diet effect: $\mathrm{F}(1,12)=243.9, p<0.0001$ ) (Table 1 ' $\mathrm{n}-6 / \mathrm{n}-3$ '). LPS treatment had no additional effect on this ratio (LPS effect: $\mathrm{F}(1,12)=1.485, \quad p=0.2465 ; \quad$ interaction: $\mathrm{F}(1,12)=1.767$, $p=0.2085$ ) (Table 1). Specifically, increase of $n-6 / n-3$ PUFA ratio was due to both a significant decrease of $n-3$ PUFAs (diet effect: $\mathrm{F}(1,12)=50.99, p<0.0001$ ) and a significant increase of $\mathrm{n}-6$ PUFAs (diet effect: $\mathrm{F}(1,12)=244.1$, $p<0.0001$ ) (Table 1 ' $n-3$ ' and ' $n-6$ '). More precisely, DHA (22:6n-3) and n-3 docosapentaenoic acid (n-3 DPA, 22:5 $\mathrm{n}-3)$ levels were significantly decreased in the hippocampus of n-3-deficient animals (two-way ANOVA with diet and treatment as factors; DHA: $\mathrm{F}(1,12)=45.14, p<0.0001 ; \mathrm{n}-3$ DPA: $F(1,12)=22.39, p=0.0005)$ (Table 1). These changes were associated with a significant increase in LA (18:2n-6), AA $(20: 4 n-6)$ and n-6 DPA $(22: 5 n-6)$ levels (two-way ANOVA with diet and treatment as factors; diet effect: LA $\mathrm{F}(1,12)=85.38, p<0.0001 ;$ AA $\mathrm{F}(1,12)=28.04, p=0.0002$; n-6 DPA $\mathrm{F}(1,12)=898.4, p<0.0001)$.

LPS treatment had no effects on n-6 and n-3 PUFA levels (LPS effect: $\mathrm{F}(1,12)=0.3390, p=0.5712$; interaction: $\mathrm{F}(1,12)=$ 2.718, $p=0.1252$ ) and composition (LPS effect: DHA $\mathrm{F}(1,12)=0.12, p=0.74 ; \mathrm{n}-3$ DPA $\mathrm{F}(1,12)=0.1, p=0.76$; EPA $\mathrm{F}(1,12)=0.16, \quad p=0.7 ; \quad$ LA $\mathrm{F}(1,12)=3.39, \quad p=0.091 ; \quad$ AA $\mathrm{F}(1,12)=0.47, p=0.50 ; \mathrm{n}-6$ DPA $\mathrm{F}(1,12)=3.65, p=0.08)$.

We found a significant interaction between LPS treatment and diet for n-3 PUFAs and for n-6 DPA (n-3 PUFAs: interaction: $\mathrm{F}(1,12)=5.22, p=0.0413$; LPS effect: $\mathrm{F}(1,12)=$ $0.1395, p=0.7153 ; \mathrm{n}-6$ DPA: $\mathrm{F}(1,12)=6.02, p=0.03 ; \mathrm{LA}$ $\mathrm{F}(1,12)=0.11, p=0.75$; AA F $(1,12)=2.24, p=0.16)$ : the decrease in total n-3 PUFAs and the increase in n-6 DPA were higher for the LPS-treated mice than for the saline-treated mice.

Other fatty acids. We also measured 20:3 n-9, total saturated fatty acid, total PUFA, and total monounsaturated fatty acid levels in the hippocampus of n-3-deficient animals compared with n-3-balanced mice. While saturated fatty acid levels were similar in all groups, monounsaturated fatty acids and 20:3 n-9 levels were significantly decreased $\mathrm{n}$-3-deficient animals compared with n-3-balanced mice. (Two-way ANOVA with diet and treatment as factors; diet effect: $20: 3 \mathrm{n}-9 \mathrm{~F}(1,12)=179.9, p<0.0001$; monounsaturated fatty acid $\mathrm{F}(1,12)=10.75, p=0.007$ ) (Table 1 ). Total PUFA levels were not significantly different between groups but we found a significant interaction between diet and treatment (two-way ANOVA with diet and treatment as factors; interaction: $\mathrm{F}(1,12)=6.55, p=0.025)$.

Overall, our data showed that decreasing the levels of n-3 PUFAs in the diet for 2 months starting at post-natal day 21 was sufficient to significantly unbalance the hippocampal $\mathrm{n}-6 / \mathrm{n}-3$ PUFA ratio, towards more $\mathrm{n}-6$ PUFAs in the form of LA, AA, and DPA n-6 and less n-3 PUFAs in the form of DHA and DPA n-3.

\footnotetext{
Figure 2 LPS treatment impairs LTD in the hippocampus of n-3 PUFA-deficient mice and decreases spine density of hippocampal neurons in both n-3 PUFA-balanced and n-3 PUFA-deficient mice. (a) High frequency stimulation (HFS) of CAI stratum radiatum layer induced a long-term potentiation (LTP) of evoked fEPSP recorded in CAI stratum radiatum layer, in n-3 PUFA-balanced mice injected with either saline ( $n=13$ slices in 10 mice, white symbols) or LPS ( $n=16$ slices in 14 mice, grey symbols), as well as in n-3 PUFA-deficient mice injected with either saline $(n=18$ slices in 16 mice, black symbols) or LPS ( $n=16$ slices in 14 mice, grey symbols). Average time courses of mean fEPSP were normalized to baseline. Low frequency stimulation (LFS) of CAI stratum radiatum layer induced a long-term depression (LTD) of evoked fEPSP recorded in CAI stratum radiatum layer, in n-3 PUFA-balanced mice injected with either saline ( $n=13$ slices in 12 mice, white symbols) or LPS ( $n=8$ slices in 8 mice, grey symbols), as well as in $n-3$ PUFA-deficient mice injected with saline ( $n=7$ slices in 7 mice, black symbols). However, LTD was impaired in LPS-treated $n-3$ PUFA-deficient mice ( $n=7$ slices in 7 mice, grey symbols). Average time courses of mean fEPSP were normalized to baseline $(* P<0.05)$. Last panel represents fEPSP (as a percentage of the baseline) 45 min after the protocol of stimulation. Data are expressed as mean $\pm \mathrm{SEM} * p<0.05$, ** $p<0.01$, paired $t$-test between baseline and mean normalized fEPSP 45 min post induction); (b) Left panel: representative images of Golgi staining from all conditions, scale bar $=10 \mu \mathrm{m}$. Right panel: Quantification of the number of spines on dendritic trees of pyramidal neurons of the hippocampus (CAI region). Data are presented as the number of spines per $10 \mu \mathrm{m}$. The first two graphs show the average number of spines on the apical and basal dendrites respectively. N-3 PUFA-deficient/saline: $n=3, n-3$ PUFA-deficient/LPS: $n=3, n-3$ PUFA-balanced/saline $n=3, n-3$ PUFA-balanced/LPS $n=5$.
} 
LPS Treatment Induces Spatial Memory Impairment in n-3 PUFA-Deficient Mice

Because inflammation and nutritional manipulations are both known to interfere with spatial memory (Yirmiya and Goshen, 2011; Moranis et al, 2012; Delpech et al, 2015a), we evaluated spatial memory performances using a Y-maze test with a 30-min of inter-trial interval in mice fed an n-3 PUFA-deficient or -balanced diet (Figure 1). A three-way ANOVA analysis revealed a significant effect of novelty $(\mathrm{F}(1,49)=43.33, \quad p<0.0001)$ and a significant interaction between arm, treatment, and $\operatorname{diet}(\mathrm{F}(2,49)=4.47$, $p=0.017$ ), with no significant effect of diet alone or treatment alone (diet effect $\mathrm{F}(2,49)=0.45, p=0.64$; LPS effect $\mathrm{F}(1,49)=0.18, p=0.67)$. Post hoc analysis revealed

a

Long-term plasticity

$\mathrm{n}-3$ balanced diet
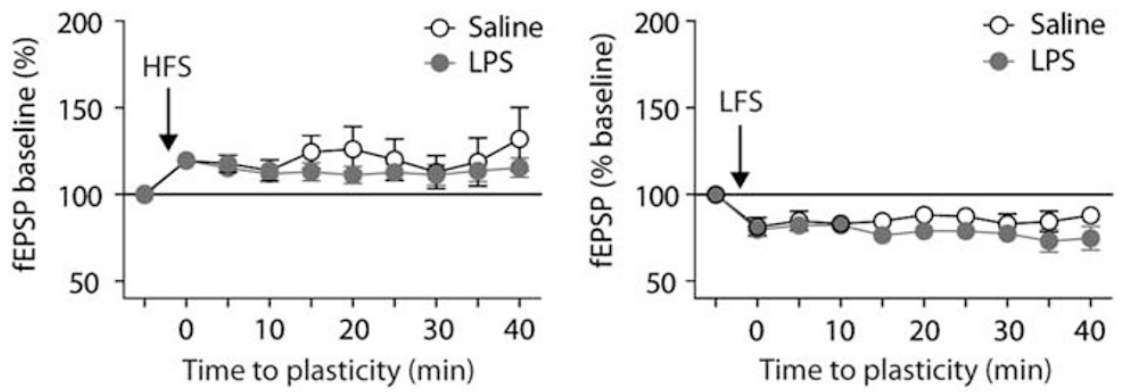

$\mathrm{n}$-3 deficient diet
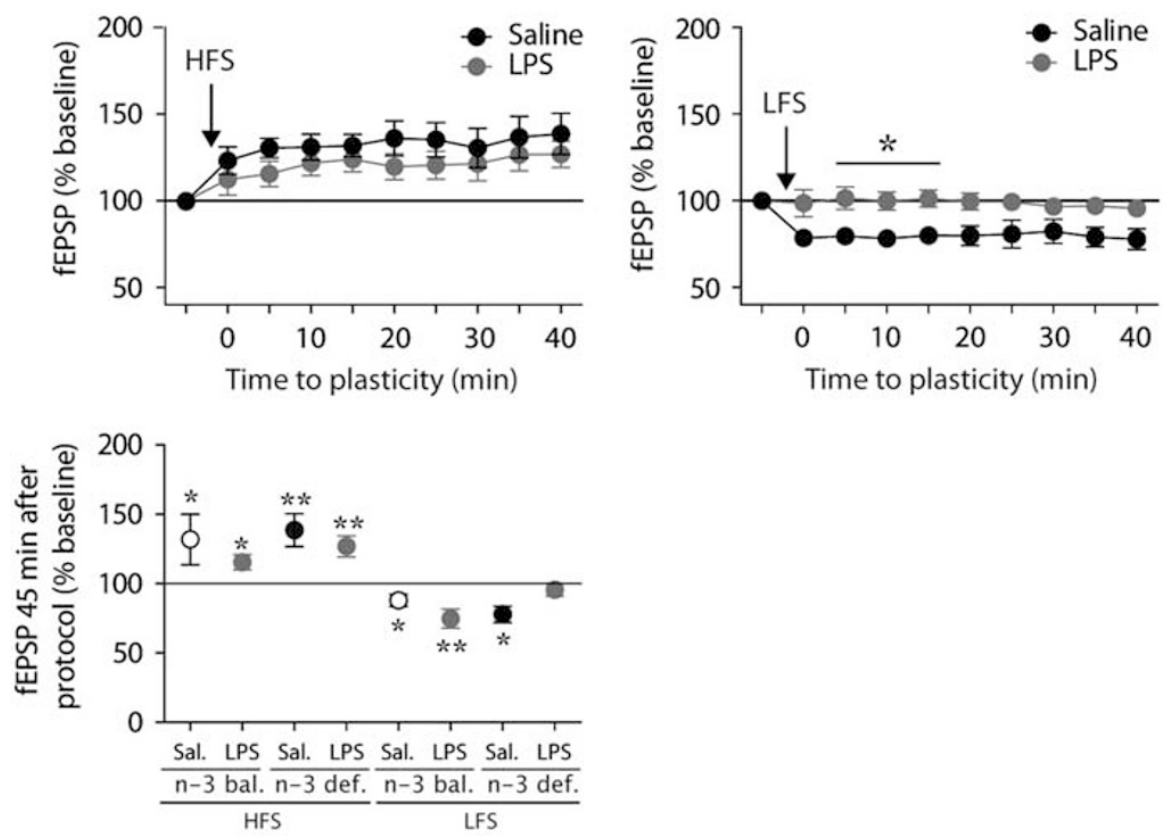

b
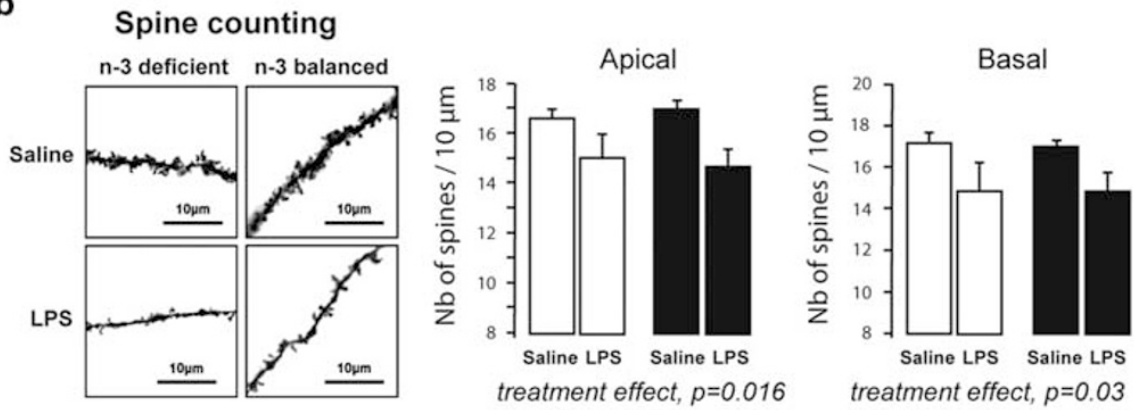

$\mathrm{n}-3$ balanced diet

n-3 deficient diet 
that both n-3 PUFA-balanced and n-3 PUFA-deficient mice spent more time in the novel arm compared with the familiar one after saline treatment $(p<0.01)$ (Figure 1$)$. However, only n-3 PUFA-balanced mice significantly distinguished between the novel and the familiar arm after LPS treatment $(p<0.01)$, while n-3 PUFA-deficient mice exhibited a random exploration of the two arms $(p=0.72)$. Thus, our behavioral data suggested that dietary n-3 deficiency increased vulnerability to LPS-induced spatial memory impairment.

\section{LPS Treatment Impairs LTD in the Hippocampus of n-3 PUFA-Deficient Mice}

To correlate behavioral observations with neuronal activity, we measured long-term synaptic plasticity using ex vivo electrophysiology in the CA1 region of the dorsal hippocampus known to be involved in spatial memory (Goshen et al, 2011) (Figure 2a). Basal synaptic excitability was first assessed by plotting fEPSP amplitude against stimulation intensity (input/output curves). We could not find any significant difference between mice fed either diet (data not shown). We next investigated paired pulse ratio as a marker of presynaptic short-term plasticity (Katz and Miledi, 1968). We evoked paired fEPSP with intervals ranging from 25 to $500 \mathrm{~ms}$ and we observed paired pulse facilitation, with greater amplitude of fEPSP2 compared with fEPSP1. The ratio fEPSP2/fEPSP1 did not differ between mice fed with n-3 PUFA-balanced diet or n-3 PUFA-deficient diet, suggesting no change in release probability (n-3 PUFAbalanced diet $(n=4)$ vs n-3 PUFA-deficient diet $(n=12)$, respectively, $25 \mathrm{~ms}: 2.18 \pm 0.54$ vs $1.78 \pm 0.12$; $50 \mathrm{~ms}: 2.02 \pm$ 0.43 vs $1.62 \pm 0.06 ; 100 \mathrm{~ms}: 1.43 \pm 0.08$ vs $1.43 \pm 0.03 ; 200 \mathrm{~ms}$ : $1.22 \pm 0.05$ vs $1.17 \pm 0.05 ; 300 \mathrm{~ms}: 1.11 \pm 0.02$ vs $1.10 \pm$ 0.03 ; $500 \mathrm{~ms}: 1.05 \pm 0.01$ vs $1.07 \pm 0.01$; $p>0.05$, MannWhitney test).

On each experimental group, we then performed HFS and LFS protocols to elicit long-term potentiation (LTP) and LTD, respectively (Figure 2a). In animals under n-3 PUFAbalanced diet, HFS elicited LTP with a significant increase of fEPSP amplitude compared with baseline $(99.6 \pm 0.2 \%$ baseline vs $131.9 \pm 18.3 \% 45 \mathrm{~min}$ after LTP induction; paired $t$-test, $p=0.0327$ ) (Figure $2 \mathrm{a}$ ). This response was not affected by LPS treatment $24 \mathrm{~h}$ prior to recordings $(100.3 \pm 0.5 \%$ baseline vs $115.4 \pm 5.5 \%$; $p=0.0170$ ). In $n-3$ PUFA-deficient animals, LTP was also significantly induced by HFS protocol $(99.7 \pm 0.2 \%$ baseline $v s 138.6 \pm 11.9 \% ; p=0.0044)$ and LPS injection did not alter this effect $(99.9 \pm 0.1 \%$ baseline $v s$ $126.8 \pm 7.6 \% ; p=0.0031)$. It should be noted that LTP magnitude was the same in both n-3 PUFA-balanced diet and n-3 PUFA-deficient mice (Mann-Whitney test, $p=0.3213)$.

When applying LFS protocol in n-3 PUFA-balanced animals, we observed a significant decrease of fEPSPs compared with baseline $(99.9 \pm 0.1 \%$ baseline vs $87.9 \pm$ $4.4 \% ; p=0.0134)$ that was not affected by LPS injection $(99.9 \pm 0.1 \%$ baseline vs $74.8 \pm 6.8 \% ; p=0.0077)$. In salinetreated n-3 PUFA-deficient animals, LFS protocol also induced a significant LTD $(99.9 \pm 0.1 \%$ baseline $v s 77.8 \pm$ $6.0 \%, p=0.0101$ ) that was not different from LTD in $\mathrm{n}-3$ PUFA-balanced animals (Mann-Whitney test, $p=0.8357$ ). Remarkably, we observed that LPS treatment $24 \mathrm{~h}$ prior to recordings completely prevented LTD induction in n-3 PUFA-deficient animals following LFS protocol (100.0 \pm $0.1 \%$ baseline $v s 95.6 \pm 4.6 \%, p=0.3750$ ) (Figure $2 \mathrm{a}$ ).

\section{LPS Treatment Decreases Spine Density in the Hippocampus of Both n-3 PUFA-Balanced and n-3 PUFA-Deficient Mice}

We then quantified dendritic arborization and spine density on basal and apical arborizations of pyramidal neurons in the CA1 region of the dorsal hippocampus. No effect of diet nor treatment was revealed on dendritic arborization (data not shown). However, a 24-h LPS treatment significantly decreased the number of spines on both side (basal and apical) of CA1 neurons (Figure 2b). PUFA dietary intervention had no effect on spine density whatever the arborization considered (two-way ANOVA with diet and treatment as factors; treatment effect, apical arborization $\mathrm{F}(1,10)=8.3$, $p=0.016$; basal arborization $\mathrm{F}(1,10)=5.9, p=0.03$; diet effect: apical arborization $\mathrm{F}(1,10)=0.09, p=0.77$; basal arborization $\mathrm{F}(1,10)=0.07 ; p=0.8$; interaction: apical arborization $\mathrm{F}(1,10)=0.07, \quad p=0.8 ;$ basal arborization $\mathrm{F}(1,10)=0.003$; $p=0.96)$.

\section{N-3 PUFA Deficiency Alters LPS-Induced Hippocampal Production of Cytokines}

Both cytokines and chemokines produced by the brain in response to an immune challenge are known to control neuronal function and memory (Hanisch and Kettenmann, 2007; Yirmiya and Goshen, 2011). Because we found that spatial memory and LTD were altered in LPS-treated n-3 PUFA-deficient mice only, we thus quantified the hippocampal levels of pro-inflammatory cytokines. Our data showed that LPS induced pro- and anti-inflammatory cytokine mRNA synthesis in both diet groups. However, the extent of the induction was significantly lower in the hippocampus of n-3 PUFA-deficient mice compared with the n-3 PUFA-balanced group over time (Figure 3a for LPS/saline ratio and Table 2 for raw data). The effect was the most obvious $2 \mathrm{~h}$ post LPS injection (at $2 \mathrm{~h}, p<0.001$ for IL- $1 \beta$, IL-6 and TNF- $\alpha$ and $p<0.05$ for IL-10; two-way ANOVA with time and diet/treatment as factors; time effect, IL- $1 \beta \mathrm{F}(3,62)=29.59, p<0.0001$; IL-6 $\mathrm{F}(3,64)=32.60$, $p<0.0001$; TNF- $\alpha \mathrm{F}(3,66)=16.72, p<0.0001$; IL-10 F $(3,62)=$ 24.60, $p<0.0001$; LPS/diet effect: IL- $1 \beta \quad \mathrm{F}(3,62)=43.71$, $p<0.0001$; IL-6 F $(3,64)=14.26, p<0.0001$; TNF- $\alpha \mathrm{F}(3,66)=$ 38.52, $p<0.0001$; IL-10 $\mathrm{F}(3,62)=12.00, p<0.0001$; interaction: IL- $1 \beta \mathrm{F}(9,62)=16.34, p<0.0001$; IL-6 $\mathrm{F}(9,64)=19.09$, $p<0.0001$; TNF- $\alpha \mathrm{F}(9,66)=11.02, p<0.0001$; IL-10 F $(9,62)=$ $8.43, p<0.0001)$.

In addition, like for the cytokines, CXCL10 mRNA expression was significantly lower in the hippocampus of n-3 PUFA-deficient mice compared with n-3 PUFAbalanced mice $2 \mathrm{~h}$ post LPS treatment (two-way ANOVA with time and diet/treatment as factors; at $2 \mathrm{~h}, \mathrm{n}-3$ PUFA-deficient $v s$ n-3 PUFA-balanced, $p<0.001$; time effect, $\mathrm{F}(3,65)=31.51, \quad p<0.0001 ; \quad$ LPS/diet effect: $\mathrm{F}(3,65)=$ 14.40, $p<0.0001$; interaction: $\mathrm{F}(9,65)=10.56, p<0.0001)$ (Figure 3b).

We finally measured mRNA levels of GPR84, a lipid receptor that has been recently identified as microglia- 

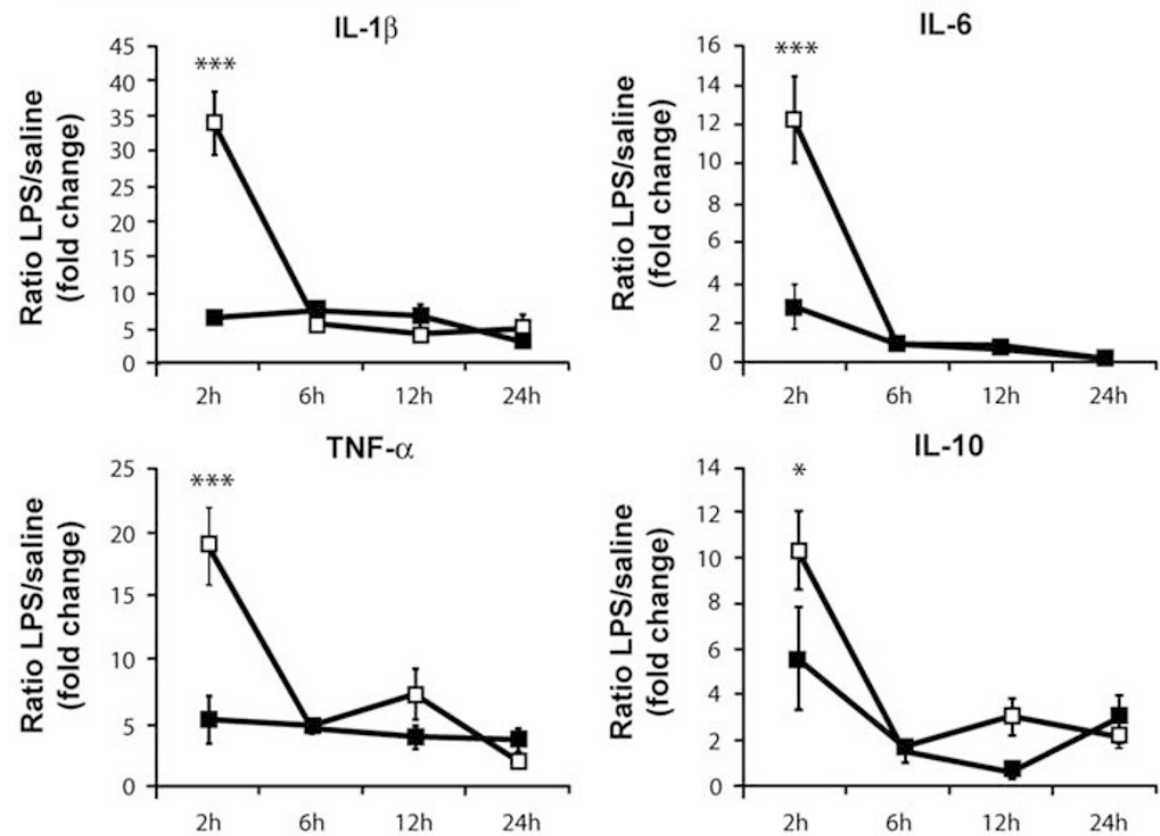

b CXCL10 mRNA expression
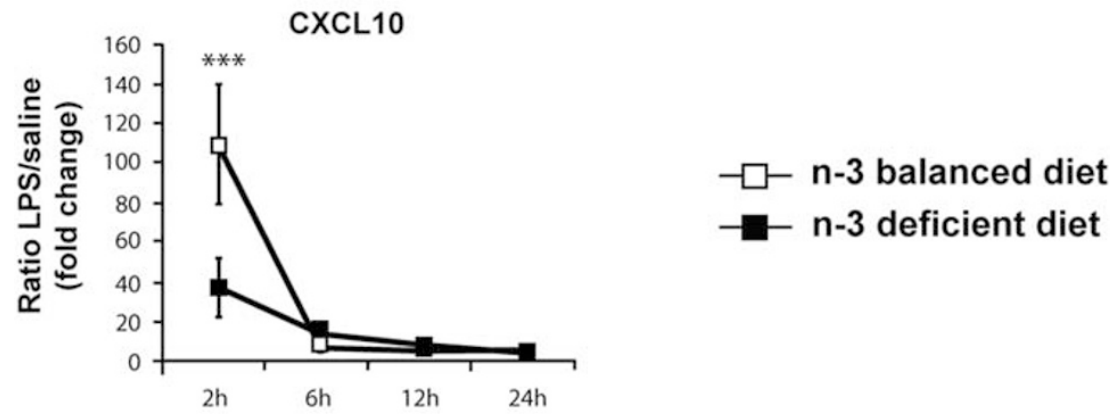

C

GPR 84 mRNA expression GPR 84

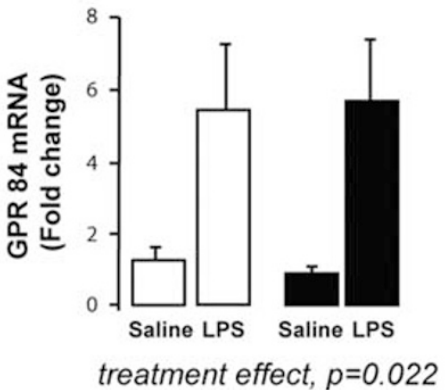

Figure 3 N-3 PUFA deficiency alters LPS-induced hippocampal production of cytokines. (a) Kinetics of expression of IL- I $\beta$, IL-6, TNF- $\alpha$, and IL- I0 mRNA in response to saline or LPS injection (ratio LPS/saline, arbitrary unit). Data are expressed as mean \pm SEM (n-3 PUFA-deficient vs n-3 PUFA-balanced mice, * $p<0.05$; **** $p<0.00$ I). $n=4-6$ mice per goup; (b) Kinetics of expression of CXCLI 0 mRNA in response to saline or LPS injection (ratio LPS/saline, arbitrary unit). Data are expressed as mean \pm SEM (n-3 PUFA-deficient vs $n-3$ PUFA-balanced mice, **** $p<0.00 I$ ). $n=4-6$ mice per goup. (c) GPR 84 mRNA expression in the hippocampus $24 \mathrm{~h}$ post saline or LPS injection. Data are expressed as mean \pm SEM (treatment effect, $* *$ $p=0.0022$ ). $n=4-5$ mice per group.

specific (Audoy-Remus et al, 2015). While LPS injection significantly increased GPR 84 mRNA expression, we could not find any difference between diets $24 \mathrm{~h}$ post LPS (Figure 3c) suggesting that the number of microglia does not vary in our paradigm (two-way ANOVA with diet and treatment as factors: diet effect, $\mathrm{F}(1,15)=0.002, p=0.96$; treatment effect: $\mathrm{F}(1,15)=13.54, \quad p=0.0022$; interaction: $\mathrm{F}(1,15)=0.06, p=0.81)$. These data confirmed our previous 
Table 2 mRNA Expression of Cytokines in the Hippocampus

\begin{tabular}{|c|c|c|c|c|c|}
\hline & \multicolumn{2}{|c|}{ n-3-deficient diet } & \multicolumn{2}{|c|}{ n-3-balanced diet } & \\
\hline & Saline & LPS & Saline & LPS & \\
\hline \multicolumn{6}{|l|}{$\overline{I L-6}$} \\
\hline $2 \mathrm{~h}$ & $1.11 \pm 0.27$ & $3.17 \pm 1.26$ & $1.02 \pm 0.09$ & $12.58 \pm 2.23$ & \#\#\# \\
\hline $6 \mathrm{~h}$ & $1.02 \pm 0.06$ & $0.92 \pm 0.08$ & $1.00 \pm 0.04$ & $0.91 \pm 0.07$ & \\
\hline $12 \mathrm{~h}$ & $1.04 \pm 0.17$ & $0.90 \pm 0.09$ & $1.01 \pm 0.08$ & $0.70 \pm 0.17$ & \\
\hline $24 \mathrm{~h}$ & $0.84 \pm 0.05$ & $0.15 \pm 0.02$ & $1.02 \pm 0.09$ & $0.20 \pm 0.03$ & \\
\hline \multicolumn{6}{|l|}{$\| L-\mid \beta$} \\
\hline $2 \mathrm{~h}$ & $1.66 \pm 0.74$ & $11.21 \pm 5.20$ & $1.06 \pm 0.19$ & $36.29 \pm 4.81$ & \#\#\# \\
\hline $6 \mathrm{~h}$ & $0.89 \pm 0.17$ & $7.12 \pm 0.44$ & $1.08 \pm 0.22$ & $6.23 \pm 0.43$ & \\
\hline $12 \mathrm{~h}$ & $0.66 \pm 0.06$ & $4.61 \pm 1.09$ & $1.04 \pm 0.15$ & $4.52 \pm 0.27$ & \\
\hline $24 \mathrm{~h}$ & $1.12 \pm 0.15$ & $3.73 \pm 0.23$ & $1.03 \pm 0.13$ & $5.35 \pm 1.25$ & \\
\hline \multicolumn{6}{|l|}{ TNF- $\alpha$} \\
\hline $2 \mathrm{~h}$ & $1.20 \pm 0.22$ & $6.38 \pm 2.27$ & $1.01 \pm 0.07$ & $19.06 \pm 3.07$ & \#\#\# \\
\hline $6 \mathrm{~h}$ & $0.95 \pm 0.11$ & $4.65 \pm 0.34$ & $1.09 \pm 0.20$ & $5.00 \pm 0.42$ & \\
\hline $12 \mathrm{~h}$ & $0.75 \pm 0.12$ & $5.46 \pm 1.46$ & $1.04 \pm 0.13$ & $4.10 \pm 0.52$ & \\
\hline $24 \mathrm{~h}$ & $0.97 \pm 0.15$ & $1.98 \pm 0.34$ & $1.00 \pm 0.05$ & $3.74 \pm 0.83$ & \\
\hline \multicolumn{6}{|l|}{$1 \mathrm{~L}-10$} \\
\hline $2 \mathrm{~h}$ & $1.41 \pm 0.81$ & $7.87 \pm 3.15$ & $1.37 \pm 0.52$ & $14.23 \pm 2.35$ & \#\#\# \\
\hline $6 \mathrm{~h}$ & $0.69 \pm 0.13$ & $1.18 \pm 0.15$ & $1.08 \pm 0.16$ & $1.60 \pm 0.52$ & \\
\hline $12 \mathrm{~h}$ & $0.57 \pm 0.11$ & $1.74 \pm 0.46$ & $1.23 \pm 0.43$ & $0.70 \pm 0.25$ & \\
\hline $24 \mathrm{~h}$ & $1.62 \pm 0.47$ & $3.41 \pm 0.73$ & $1.01 \pm 0.06$ & $2.98 \pm 1.06$ & \\
\hline
\end{tabular}

Although LPS treatment significantly induced cytokines production in both diet groups, only statistics for ' $n$-3-deficient LPS vs n-3-balanced LPS' appear in the table for clarity sake, see \#.

results showing that neither dietary n-3 PUFA deficiency nor transgenic increase in n-3 PUFA modify the number of microglial cells in the hippocampus (Madore et al, 2014; Delpech et al, 2015a).

\section{LPS Treatment Similarly Increases Cytokine Release, But Not Corticosterone in Plasma of Both n-3 PUFA-Balanced and n-3 PUFA-Deficient Mice}

The production of brain pro-inflammatory cytokines in response to an immune challenge relies on the peripheral synthesis of inflammatory factors by the innate immune system (Dantzer et al, 2008).

We then analyzed the time course of plasma cytokine release at $2,6,12$, and $24 \mathrm{~h}$ post LPS treatment (Figure 4 ). The pro-inflammatory cytokines IL- $1 \beta$, IL- 6 , and TNF- $\alpha$ as well as the anti-inflammatory cytokine IL-10 were all significantly increased $2 \mathrm{~h}$ post immune challenge independently on the diet $(p<0.001)$ (Figure $4 a)$. Cytokine plasma levels followed the same kinetics of expression after LPS or saline treatment whatever the diet considered (two-way ANOVA with time and diet/treatment as factors; time effect: IL-1 $\beta \quad \mathrm{F}(3,71)=12.80, \quad p<0.0001 ; \quad$ IL-6 $\quad \mathrm{F}(3,74)=90.12$, $p<0.0001 ; \quad$ TNF- $\alpha \quad \mathrm{F}(3,80)=32.90, \quad p<0.0001 ; \quad$ IL-10 $\mathrm{F}(3,79)=23.03, p<0.0001$; LPS/diet effect: IL- $1 \beta \mathrm{F}(3,71)=$ $24.74, p<0.0001$; IL-6 F $(3,74)=37.95, p<0.0001 ;$ TNF- $\alpha$ $\mathrm{F}(3,80)=13.98, p<0.0001$; IL-10 $\mathrm{F}(3,79)=16.70, p<0.0001$; interaction: IL- $1 \beta \mathrm{F}(9,71)=5.643, p<0.0001$; IL-6 F $(9,74)=$ $31.23, p<0.0001$; TNF- $\alpha \mathrm{F}(9,80)=10.76, p<0.0001$; IL-10 $\mathrm{F}(9,79)=7.367, \quad p<0.0001)$. Only IL- $1 \beta$ at $6 \mathrm{~h}$ was found to be significantly more expressed in LPS-treated n-3 PUFA-deficient animals compared with LPS-treated n-3 PUFA-balanced mice (n-3 PUFA-deficient/LPS $v s$ n-3 PUFA-balanced LPS, $p<0.01)$. LPS-induced peripheral cytokine production was accompanied by a significant decrease in the weight of mice at $24 \mathrm{~h}$ independently on the diet (two-way ANOVA with diet and treatment as factors; diet effect $\mathrm{F}(1,35)=0.147, p=0.7033$; LPS effect $\mathrm{F}(1,35)=$ $111.12, p<0.0001$; interaction $\mathrm{F}(1,35)=1.237, p=0.2737)$. (Figure $4 \mathrm{~b}$ ). This weight loss was paralleled by a significant decrease in food intake after LPS injection in both groups (two-way ANOVA with diet and treatment as factors; diet effect $\mathrm{F}(1,35)=0.132, p=0.7183$; LPS effect $\mathrm{F}(1,35)=85.969$, $p<0.0001$; interaction $\mathrm{F}(1,35)=3.806, p=0.0591)$.

In addition, levels of corticosterone, a well-known modulator of peripheral and brain cytokine production (Bellavance and Rivest, 2014), were measured in the blood of n-3 PUFA-deficient and -balanced mice 2, 6, 12, and $24 \mathrm{~h}$ after saline or LPS treatment (Figure 4c). Corticosterone levels were the same in saline-treated animals whatever the diet considered. Only at $6 \mathrm{~h}$ post saline injection, we could detect a slight but significant difference between $n-3$ PUFA-deficient and n-3 PUFA-balanced animals $(p<0.05)$. In response to LPS injection, corticosterone levels were significantly higher in the blood of n-3 PUFA-deficient mice compared with n-3 PUFA-balanced mice (two-way ANOVA with time and diet/treatment as factors; time effect, $\mathrm{F}(3,48)=$ 134.5, $p<0.0001$; diet/treatment effect, $F(3,48)=45.43$, $p<0.0001$; interaction, $\mathrm{F}(9,48)=21.38, p<0.0001$; at $2 \mathrm{~h}$ post LPS injection, n-3 PUFA-deficient/LPS $v s$ n-3 PUFAbalanced LPS: $p<0.0001$ ).

\section{DISCUSSION}

Overall, our data showed that a 2-month exposure to an n-3 PUFA-deficient diet was sufficient to significantly unbalance hippocampal n-6/n-3 PUFA ratio, towards more n-6 PUFAs and less n-3 PUFAs. These changes in hippocampal fatty acid contents increased vulnerability of these mice to develop spatial memory alteration in response to LPS. Decreased spine density in the hippocampus and increased plasma cytokine production were similar in both n-3 PUFAdeficient and -balanced mice in response to LPS. On the opposite, long-term synaptic plasticity, namely LTD, together with cytokine and chemokine production was attenuated only in the hippocampus of n-3 PUFA-deficient mice after LPS treatment. In addition, plasma corticosterone levels were higher. All together, our results suggested that n-3 deficiency increases the vulnerability of mice to LPS-induced memory impairment through specific alteration of synaptic plasticity and cytokines/chemokines production in the hippocampus.

To evaluate the potentiality of a 2-month diet exposure to modulate spatial memory and inflammatory processes, we fed mice with n-3 PUFA-deficient $v s$-balanced diet for 2 months, starting after the lactation phase, and quantified hippocampal PUFAs contents. The role of dietary n-3 PUFAs in cognitive function has already been extensively 
a

Plasma cytokines
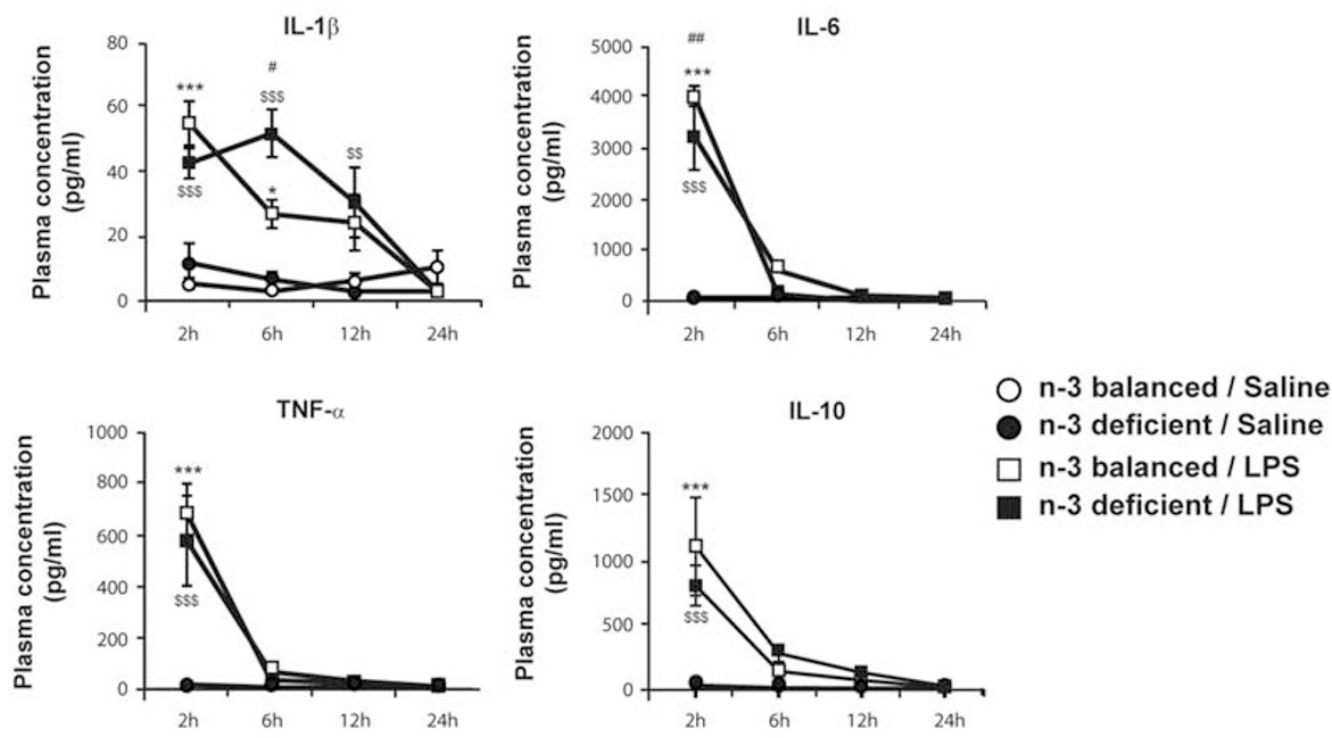

b

Sickness behavior
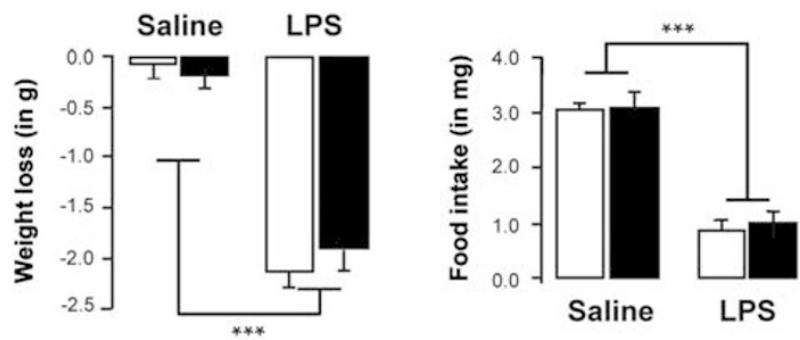

$\square \mathrm{n}-3$ balanced diet

Blood corticosterone
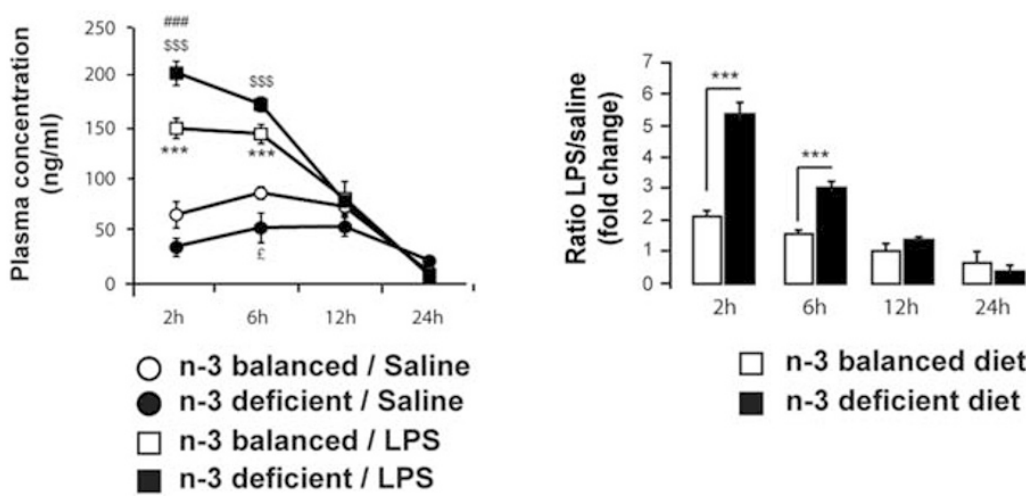

$\square \mathrm{n}-3$ balanced diet

$\square$ n-3 deficient diet

Figure 4 LPS treatment similarly induces sickness behavior and cytokine release, but not corticosterone, in the plasma of $n-3$ PUFA balanced and n-3 PUFA-deficient mice. (a) Plasma concentration of the pro-inflammatory cytokines IL- I $\beta, \mathrm{IL}-6$, and TNF- $\alpha$ and the anti-inflammatory cytokine IL- 0 in response to saline or LPS injection (in pg/ml). Measures were performed 2, 6, 12, and $24 \mathrm{~h}$ post treatment. Data are expressed as mean \pm SEM. * $\mathrm{n}$-3 PUFA-balanced/ saline vs n-3 PUFA-balanced/LPS; ${ }^{\$}$ n-3 PUFA-deficient/saline vs n-3 PUFA-deficient/LPS, ${ }^{\#}$ n-3 PUFA-deficient/LPS vs n-3 PUFA-balanced/LPS (*., $p<0.05$; $\$ \$, \# \# p<0.01$; ****,\$\$. \#\#\# $p<0.00$ I). $n=5-6$ mice per group; (b) Weight loss and food intake of mice fed an $n$-3 PUFA-deficient or $n$-3 PUFA-balanced diet $24 \mathrm{~h}$ after saline or LPS treatment. All mice developed a sickness behavior of same amplitude in response to LPS injection. Data are expressed as mean \pm SEM (**** $<0.00$ I). $n=6$ per group; (c) Left panel: Plasma concentration of corticosterone in response to saline or LPS in n-3 PUFA-deficient and $n-3$ PUFAbalanced animals (hormonal concentration expressed as $\mathrm{ng} / \mathrm{ml}$ ). Measures were performed 2, 6, 12 , and $24 \mathrm{~h}$ post treatment. Data are expressed as mean \pm SEM. * $n-3$ PUFA-balanced/saline vs $n$-3 PUFA-balanced/LPS; ${ }^{\$} n-3$ PUFA-deficient/saline vs $n$-3 PUFA-deficient/LPS, ${ }^{\#} n$-3 PUFA-deficient/LPS vs $n-3$ PUFA-balanced LPS, ${ }^{\epsilon} n-3$ PUFA-deficient/saline vs n-3 PUFA-balanced/saline $\left({ }^{\epsilon} p<0.05\right.$; $* * * * * \$ \$, \# \# \#<0.00$ I); Right panel: LPS/saline ratio of corticosterone concentration (***** $p<0.00 \mathrm{I}) . n=5$ mice per group. 
studied either during the perinatal period (the most sensitive period in terms of brain development and lipid accretion), or in aging (a period of high vulnerability for cognitive decline and dementia) (Luchtman and Song, 2013; Joffre et al, 2014). In most of animal models, brain n-3 PUFA deficiency is induced by feeding an n-3 PUFA-deficient diet throughout life for two to three generations (Gamoh et al, 1999; Moriguchi et al, 2000; Moriguchi and Salem, 2003; Su, 2010; Orr et al, 2013), and only few studies dealt with the effect of a 2-month exposure to low n-3 PUFA on cognition at adulthood (Joffre et al, 2014). Moreover, only few studies reported the brain contents in PUFAs in their experimental context making difficult the comparison with our results. The present data showed that a 2-month exposure to these diets over one single generation is sufficient to significantly modulate brain lipids. More specifically, in accordance with a previous report, DHA levels were significantly decreased in n-3 PUFA-deficient animals compared with n-3 PUFAbalanced mice, while we found an increase in n-6 PUFA contents, especially of DPA n-6 and AA (Lalancette-Hebert et al, 2011).

Many studies indicate that hippocampal n-3 PUFA levels modulate cognitive performances (Joffre et al, 2014). We and others have previously shown that increased brain content of DPA $n-6$, together with reduced brain DHA content, is associated with behavioral impairment in adult rodents fed with diet deprived of n-3 PUFAs (Lim et al, 2005a; Mingam et al, 2008; Lafourcade et al, 2011; Igarashi et al, 2012; Larrieu et al, 2012; Moranis et al, 2012; Larrieu et al, 2014). Of note, in all these studies, n-3 PUFA deficiency started early in life, covering the in utero and lactation period. In the present work, mice were fed with a deficient diet starting at weaning for 2 months and did not display spatial memory impairment as measured in the Y-maze test at adulthood. Although in both experimental paradigms, we could always find the same fatty acids varying the same way, the amplitude of such modifications is different, especially for DHA (50\% decrease when diet starts on the first day of gestation $v s \quad 20 \%$ in the present study) and DPA n-6 (sevenfold increase when diet starts on the first day of gestation $v s$ fourfold increase in the present study) (Mingam et al, 2008; Lafourcade et al, 2011; Moranis et al, 2012). More studies are thus needed to understand how different lipid proportions lead to different behavioral outcomes.

The positive impact of n-3 PUFAs on cognition was not correlated to fine modifications of hippocampal neuron morphology. Indeed, PUFA dietary modulations had no effect on spine density on both basal and apical arborization. Our data are the first to evaluate the impact of a 2-month exposure to n-3 PUFA-deficient diet from pre-puberty to adulthood on neuronal architecture at adulthood. Here, we point out that the period of exposure to n-3 PUFAs might be critical as a previous work showed that DHA depletion in fetal hippocampi from E2 to E16 resulted in decrease in the number of synapses (Cao et al, 2009). Additionally, experiments performed in vitro on hippocampal neurons in culture showed that application of $1 \mu \mathrm{M}$ of DHA increased significantly the total number of synapses compared with control cultures (Kim et al, 2011). This confirmed previous results from Cao et al. (2009) showing that 10 days of in vitro supplementation with DHA at $1 \mu \mathrm{M}$ significantly promotes synaptogenesis. It would thus be very interesting to quantify the number of spines on hippocampal neurons in animals under n-3 PUFA deficiency since the first day of gestation. On the basis of the literature, one might expect a decrease in spine density in n-3 PUFA-deficient animals compared with controls.

Although the diet had no effect, LPS treatment, however, significantly decreased the number of spines in both $n-3$ PUFA-deficient and -balanced groups. A previous report already showed that spines were less stable in intraperitoneally LPS-treated mice. This was due to enhanced spine turnover so that over 8 weeks after a single LPS injection, the fraction of eliminated spines amounted to $20 \%$ of the initial population and this persistent destabilization resulted in a reduction of the total spine density (Kondo et al, 2011). However, when looking earlier, at $48 \mathrm{~h}$ after the injection, they could not find any difference in spine density (Kondo et al, 2011). Although LPS effects might be exacerbated by the nutritional context and/or the structure considered (cortex vs hippocampus), we here confirmed that a peripheral immune stimulation has an effect on spine density and highlight that n-3 PUFA deficiency does not exacerbate this effect.

Because brain inflammation and nutritional manipulations are both known to interfere with spatial memory (Cunningham and Sanderson, 2008; Su, 2010; Yirmiya and Goshen, 2011; Luchtman and Song, 2013), we evaluated spatial memory performances using a Y-maze test in mice fed an n-3 PUFA-deficient or n-3 PUFA-balanced diet. Our behavioral data demonstrated that n-3 PUFA-deficient mice were more vulnerable to LPS-induced spatial memory impairment than n-3 PUFA-balanced mice. This is in agreement with our previous studies showing that Fat-1 mice that exhibit lower $n-6 / n-3$ PUFA ratio compared with their wild-type littermates are protected from LPS-induced spatial memory deficits (Delpech et al, 2015a). Thus, the increase of hippocampal $n-6 / n-3$ PUFA ratio sensitizes the mice to the deleterious effect of LPS on memory. Interestingly, in a separate set of studies, we also showed that LPS induced alterations in hippocampal synaptic plasticity, namely LTD, whereas LTP was not affected. Recently, it has been proposed that LTD could increase the signal-tonoise ratio of a memory trace (Nicholls et al, 2008). Nicholls et al. (2008) showed that LTD could weaken previous memory traces, thereby preventing those traces from interfering with newly encoded information when the demands of a task change as it is the case in a working memory experiment (Malleret $e t a l, 2010)$. The persistence of LTD may allow acquisition of new information by restricting the body of previously stored information and suppressing interference (Malleret et al, 2010). Thus, LPS-induced inhibition of LTD in n-3 PUFA-deficient animals might be the electrophysiological basis for working memory impairment in the Y-maze. The molecular mechanisms underlying LPS-induced LTD impairment in n-3-deficient mice remain to be determined. One interesting molecular pathway is CR3 (for Complement Receptor 3) protein expressed by microglia as it has been recently demonstrated that this receptor triggers LTD in surrounding hippocampal neurons in response to LPS (Zhang et al, 2014). As n-3 PUFA deficiency influences microglia phenotype (Madore et al, 2014; Delpech et al, 2015a) and CR3 is involved in microglia priming (Ramaglia et al, 2012) and synaptic plasticity (Brennan et al, 
2012), the complement/CR3 could be a molecular intermediary of n-3 PUFA sensitization to LPS-induced synaptic activity impairment. It would then be very interesting to evaluate CR3 expression and activation under nutritional n-3 PUFA manipulation.

Animals fed an n-3 PUFA-deficient diet developed sickness behavior and cytokine release to the same extent as controls in response to LPS administration. However, the brain cytokine production was less activated in their hippocampus though still significantly increased, suggesting an uncoupling inflammatory response in $\mathrm{n}$-3-deficient mice to LPS. This is in agreement with our previous study showing an uncoupling of peripheral and central inflammatory events in n-3-deficient mice (Mingam et al, 2008). Mingam et al. (2008) demonstrated that LPS-induced IL-6 expression was not impaired but its signaling pathway (namely STAT3) was not activated in the brain of n-3 PUFA deficient mice given from gestation to 8 weeks of age. The mechanisms underlying the dichotomy between peripheral and central inflammatory responses under n-3 PUFA deficiency still need to be addressed thoroughly. However, corticosterone could be involved as this hormone, which is well known to regulate cytokine production both at the periphery and in the brain is chronically released in n-3 PUFA mice (Larrieu et al, 2014) and overproduced in response to LPS as shown here. A deeper evaluation of HPA axis activity is required to firmly conclude on this point. In addition, despite n-6 DPA does not completely replace DHA in n-3 PUFA-deficient animals (Greiner et al, 2003), its increase could be involved in the lower proinflammatory cytokine production in the brain of n-3 PUFA-deficient mice. Indeed, it was shown that $n-6$ DPA reduces inflammatory mediators in human peripheral mononuclear cells in vitro (Dangi et al, 2009; Nauroth et al, 2010). Moreover, n-6 DPA can be the precursor for resolvins, involved in dampening and resolution of inflammation in macrophages (Lim et al, 2005b; Chiu et al, 2012). Further investigations are needed to decipher whether corticosterone or n-6 DPA participates to the blunting of central inflammatory response after LPS injection. One puzzling question anyway is that the attenuated brain cytokine production did not protect from the adverse effect of LPS in n-3 PUFA-deficient mice, further reinforcing the idea that nutritional n-3 PUFAs are key components of brain response to inflammation. We and others already published that ATP, another inflammatory mediator, was more potent to induce behavioral deficits under neuroinflammation than cytokines themselves (Pascual et al, 2012; Delpech et al, 2015b).

Finally, we cannot exclude that the observed effects may be specific to adolescent period as animals were deprived from post-natal day 21 for 2 months. Indeed, a recent study demonstrated that modulating n-3 PUFA levels throughout development (both pre- and post-weaning) may increase resiliency to emotional stressors and decrease susceptibility to mood disorders that commonly arise during adolescence (Weiser et al, 2015). Another study also reported a nutritional contribution to optimal cognitive and affective functioning in adolescents. More specifically, n-3 PUFA deficiency disrupts adolescent behaviors through enhanced dorsal striatal dopamine availability (Bondi et al, 2014). Finally, in adolescents, dietary n-3 PUFA deficiency across consecutive generations produced a modality-selective and task-dependent impairment in cognitive and motivated behavior distinct from the deficits observed in adults (Rao et al, 2007).

In conclusion, the present study looked at the role of PUFAs on the effect of an immune challenge on both inflammatory response and neuronal activity using a model of post-developmental n-3 PUFA deficiency. Our results indicated that a greater hippocampal n-6/n-3 PUFA ratio was associated with LPS-induced cognitive alterations that were likely to rely on synaptic plasticity deficits suggesting that a 2-month exposure to n-3 PUFA dietary deficiency greatly sensitizes the brain to adverse immune stimuli. The results of this study not only provide insight into how lipids can influence immune response but also suggest a role for n-3 PUFAs in the prevention of spatial memory deficit linked to inflammation.

\section{FUNDING AND DISCLOSURE}

The authors declare no conflict of interest.

\section{ACKNOWLEDGMENTS}

This work was supported by INRA, Idex Bordeaux University, ANR MoodFood, Fondation pour la Recherche Médicale (FRM) and Société Française de Nutrition (SFN). JCD is the recipient of a post-doctoral fellow from Région Aquitaine. $\mathrm{CBB}$ is the recipient of the Agreenskills postdoctoral grant support. This work benefited from the facilities and expertise of the imagery platform Imag'In (www.incia.u-bordeaux1.fr), which is supported by CNRS and Région Aquitaine. We thank Dr A. Kaffman for his insightful comments and P. Birac, C. Tridon, and M. Cadet for taking care of the mice.

\section{REFERENCES}

Arsenault D, Julien C, Calon F (2011). Chronic dietary intake of alpha-linolenic acid does not replicate the effects of DHA on passive properties of entorhinal cortex neurons. Br J Nutr 107: 1099-1111.

Audoy-Remus J, Bozoyan L, Dumas A, Filali M, Lecours C, Lacroix S et al (2015). GPR84 deficiency reduces microgliosis, but accelerates dendritic degeneration and cognitive decline in a mouse model of Alzheimer's disease. Brain Behav Immun 46: 112-20.

Bazan NG, Molina MF, Gordon WC (2011). Docosahexaenoic acid signalolipidomics in nutrition: significance in aging, neuroinflammation, macular degeneration, Alzheimer's, and other neurodegenerative diseases. Annu Rev Nutr 31: 321-351.

Bazinet RP, Laye S (2014). Polyunsaturated fatty acids and their metabolites in brain function and disease. Nat Rev Neurosci 15: 771-785.

Bellavance MA, Rivest S (2014). The HPA - Immune Axis and the Immunomodulatory Actions of Glucocorticoids in the Brain. Front Immunol 5: 136.

Bondi CO, Taha AY, Tock JL, Totah NK, Cheon Y, Torres GE et al (2014). Adolescent behavior and dopamine availability are uniquely sensitive to dietary omega-3 fatty acid deficiency. Biol Psychiatry 75: 38-46.

Brennan FH, Anderson AJ, Taylor SM, Woodruff TM, Ruitenberg MJ (2012). Complement activation in the injured central nervous system: another dual-edged sword? J Neuroinflammation 9: 137.

Calder PC (2013). n-3 fatty acids, inflammation and immunity: new mechanisms to explain old actions. Proc Nutr Soc 72: 326-336. 
Cao D, Kevala K, Kim J, Moon HS, Jun SB, Lovinger D et al (2009). Docosahexaenoic acid promotes hippocampal neuronal development and synaptic function. J Neurochem 111: 510-521.

Chang PK, Khatchadourian A, McKinney RA, Maysinger D (2015). Docosahexaenoic acid (DHA): a modulator of microglia activity and dendritic spine morphology. J Neuroinflammation 12: 244.

Chiu CY, Gomolka B, Dierkes C, Huang NR, Schroeder M, Purschke $\mathrm{M}$ et al (2012). Omega-6 docosapentaenoic acid-derived resolvins and 17-hydroxydocosahexaenoic acid modulate macrophage function and alleviate experimental colitis. Inflamm Res 61: 967-976.

Coutinho AE, Chapman KE (2011). The anti-inflammatory and immunosuppressive effects of glucocorticoids, recent developments and mechanistic insights. Mol Cell Endocrinol 335: 2-13.

Cunningham C, Sanderson DJ (2008). Malaise in the water maze: untangling the effects of LPS and IL-1beta on learning and memory. Brain Behav Immun 22: 1117-1127.

Dangi B, Obeng M, Nauroth JM, Chung G, Bailey-Hall E, Hallenbeck $\mathrm{T}$ et al (2009). Metabolism and biological production of resolvins derived from docosapentaenoic acid (DPAn-6). Biochem Pharmacol 79: 251-260.

Dantzer R, O'Connor JC, Freund GG, Johnson RW, Kelley KW (2008). From inflammation to sickness and depression: when the immune system subjugates the brain. Nat Rev Neurosci 9: 46-56.

De Smedt-Peyrusse V, Sargueil F, Moranis A, Harizi H, Mongrand S, Laye S (2008). Docosahexaenoic acid prevents lipopolysaccharide-induced cytokine production in microglial cells by inhibiting lipopolysaccharide receptor presentation but not its membrane subdomain localization. J Neurochem 105: 296-307.

Delpech JC, Madore C, Joffre C, Aubert A, Kang JX, Nadjar A et al (2015a). Transgenic increase in $n-3 / n-6$ fatty acid ratio protects against cognitive deficits induced by an immune challenge through decrease of neuroinflammation. Neuropsychopharmacology 40: 525-536.

Delpech JC, Saucisse N, Aubert A, Casenave F, Sans N, Layé S et al (2015b). Microglial activation exacerbates associative taste memory through purinergic modulation of glutamatergic neurotransmission. J Neurosci 35: 3022-3033.

Farooqui AA, Horrocks LA, Farooqui T (2007). Modulation of inflammation in brain: a matter of fat. J Neurochem 101: 577-599.

Fedorova I, Salem N Jr. (2006). Omega-3 fatty acids and rodent behavior. Prostaglandins Leukot Essent Fatty Acids 75: 271-289.

Gamoh S, Hashimoto M, Sugioka K, Shahdat Hossain M, Hata N, Misawa Y et al (1999). Chronic administration of docosahexaenoic acid improves reference memory-related learning ability in young rats. Neuroscience 93: 237-241.

Goshen I, Avital A, Kreisel T, Licht T, Segal M, Yirmiya R (2009). Environmental enrichment restores memory functioning in mice with impaired IL-1 signaling via reinstatement of long-term potentiation and spine size enlargement. J Neurosci 29: 3395-3403.

Goshen I, Brodsky M, Prakash R, Wallace J, Gradinaru V, Ramakrishnan C et al (2011). Dynamics of retrieval strategies for remote memories. Cell 147: 678-689.

Goshen I, Kreisel T, Ounallah-Saad H, Renbaum P, Zalzstein Y, Ben-Hur $\mathrm{T}$ et al (2007). A dual role for interleukin-1 in hippocampal-dependent memory processes. Psychoneuroendocrinology 32: 1106-1115.

Greiner RS, Catalan JN, Moriguchi T, Salem N Jr. (2003). Docosapentaenoic acid does not completely replace DHA in n-3 FA-deficient rats during early development. Lipids 38: 431-435.

Hanisch UK, Kettenmann H (2007). Microglia: active sensor and versatile effector cells in the normal and pathologic brain. Nat Neurosci 10: 1387-1394.

Igarashi M, Kim HW, Gao F, Chang L, Ma K, Rapoport SI (2012). Fifteen weeks of dietary n-3 polyunsaturated fatty acid deprivation increase turnover of n-6 docosapentaenoic acid in rat-brain phospholipids. Biochim Biophys Acta 1821: 1235-1243.

Joffre C, Nadjar A, Lebbadi M, Calon F, Laye S (2014). n-3 LCPUFA improves cognition: the young, the old and the sick. Prostaglandins Leukot Essent Fatty Acids 91: 1-20.

Katz B, Miledi R (1968). The role of calcium in neuromuscular facilitation. J Physiol 195: 481-492.

Kim HY, Moon HS, Cao D, Lee J, Kevala K, Jun SB et al (2011). $\mathrm{N}$-Docosahexaenoylethanolamide promotes development of hippocampal neurons. Biochem J 435: 327-336.

Kondo S, Kohsaka S, Okabe S (2011). Long-term changes of spine dynamics and microglia after transient peripheral immune response triggered by LPS in vivo. Mol Brain 4: 27.

Labrousse VF, Nadjar A, Joffre C, Costes L, Aubert A, Gregoire S et al (2012). Short-term long chain omega3 diet protects from neuroinflammatory processes and memory impairment in aged mice. PLoS One 7: e36861.

Lafourcade M, Larrieu T, Mato S, Duffaud A, Sepers M, Matias I et al (2011). Nutritional omega-3 deficiency abolishes endocannabinoid-mediated neuronal functions. Nat Neurosci 14: 345-350.

Lalancette-Hebert M, Julien C, Cordeau P, Bohacek I, Weng YC, Calon $\mathrm{F}$ et al (2011). Accumulation of dietary docosahexaenoic acid in the brain attenuates acute immune response and development of postischemic neuronal damage. Stroke 42: 2903-2909.

Larrieu T, Madore C, Joffre C, Laye S (2012). Nutritional n-3 polyunsaturated fatty acids deficiency alters cannabinoid receptor signaling pathway in the brain and associated anxiety-like behavior in mice. J Physiol Biochem 68: 671-681.

Larrieu T, Hilal LM, Fourrier C, De Smedt-Peyrusse V, Sans N, Capuron $L$ et al (2014). Nutritional omega-3 modulates neuronal morphology in the prefrontal cortex along with depressionrelated behaviour through corticosterone secretion. Transl Psychiatry 4: e437.

Laye S, Parnet P, Goujon E, Dantzer R (1994). Peripheral administration of lipopolysaccharide induces the expression of cytokine transcripts in the brain and pituitary of mice. Brain Res Mol Brain Res 27: 157-162.

Laye S, Gheusi G, Cremona S, Combe C, Kelley K, Dantzer R et al (2000). Endogenous brain IL-1 mediates LPS-induced anorexia and hypothalamic cytokine expression. Am J Physiol Regul Integr Comp Physiol 279: R93-98.

Lim SY, Hoshiba J, Salem N Jr. (2005a). An extraordinary degree of structural specificity is required in neural phospholipids for optimal brain function: n-6 docosapentaenoic acid substitution for docosahexaenoic acid leads to a loss in spatial task performance. J Neurochem 95: 848-857.

Lim SY, Hoshiba J, Moriguchi T, Salem N Jr. (2005b). N-3 fatty acid deficiency induced by a modified artificial rearing method leads to poorer performance in spatial learning tasks. Pediatr Res 58: 741-748.

Luchtman DW, Song C (2013). Cognitive enhancement by omega-3 fatty acids from child-hood to old age: findings from animal and clinical studies. Neuropharmacology 64: 550-565.

Madore C, Joffre C, Delpech JC, De Smedt-Peyrusse V, Aubert A, Coste L et al (2013). Early morphofunctional plasticity of microglia in response to acute lipopolysaccharide. Brain Behav Immun 34: 151-158.

Madore C, Nadjar A, Delpech JC, Sere A, Aubert A, Portal C et al (2014). Nutritional n-3 PUFAs deficiency during perinatal periods alters brain innate immune system and neuronal plasticity-associated genes. Brain Behav Immun 41: 22-31.

Malleret G, Alarcon JM, Martel G, Takizawa S, Vronskaya S, Yin D et al (2010). Bidirectional regulation of hippocampal long-term synaptic plasticity and its influence on opposing forms of memory. J Neurosci 30: 3813-3825.

Mingam R, Moranis A, Bluthe RM, De Smedt-Peyrusse V, Kelley $\mathrm{KW}$, Guesnet $\mathrm{P}$ et al (2008). Uncoupling of interleukin-6 from its 
signalling pathway by dietary n-3-polyunsaturated fatty acid deprivation alters sickness behaviour in mice. Eur J Neurosci 28: $1877-1886$.

Moranis A, Delpech JC, De Smedt-Peyrusse V, Aubert A, Guesnet P, Lavialle $M$ et al (2012). Long term adequate n-3 polyunsaturated fatty acid diet protects from depressive-like behavior but not from working memory disruption and brain cytokine expression in aged mice. Brain Behav Immun 26: 721-731.

Moriguchi T, Salem N Jr. (2003). Recovery of brain docosahexaenoate leads to recovery of spatial task performance. J Neurochem 87: 297-309.

Moriguchi T, Greiner RS, Salem N Jr (2000). Behavioral deficits associated with dietary induction of decreased brain docosahexaenoic acid concentration. J Neurochem 75: 2563-2573.

Nauroth JM, Liu YC, Van Elswyk M, Bell R, Hall EB, Chung G et al (2010). Docosahexaenoic acid (DHA) and docosapentaenoic acid (DPAn-6) algal oils reduce inflammatory mediators in human peripheral mononuclear cells in vitro and paw edema in vivo. Lipids 45: 375-384.

Nicholls RE, Alarcon JM, Malleret G, Carroll RC, Grody M, Vronskaya S et al (2008). Transgenic mice lacking NMDARdependent LTD exhibit deficits in behavioral flexibility. Neuron 58: 104-117.

Orr SK, Bazinet RP (2008). The emerging role of docosahexaenoic acid in neuroinflammation. Curr Opin Investig Drugs 9: 735-743.

Orr SK, Trepanier MO, Bazinet RP (2013). n-3 Polyunsaturated fatty acids in animal models with neuroinflammation. Prostaglandins Leukot Essent Fatty Acids 88: 97-103.

Parkhurst CN, Yang G, Ninan I, Savas JN, Yates JR 3rd, Lafaille JJ et al (2013). Microglia promote learning-dependent synapse formation through brain-derived neurotrophic factor. Cell 155: 1596-1609.

Pascual O, Ben Achour S, Rostaing P, Triller A, Bessis A (2012). Microglia activation triggers astrocyte-mediated modulation of excitatory neurotransmission. Proc Natl Acad Sci USA 109: E197-205.
Ramaglia V, Hughes TR, Donev RM, Ruseva MM, Wu X, Huitinga I et al (2012). C3-dependent mechanism of microglial priming relevant to multiple sclerosis. Proc Natl Acad Sci USA 109: 965-970.

Rao JS, Ertley RN, DeMar JC Jr., Rapoport SI, Bazinet RP, Lee HJ (2007). Dietary n-3 PUFA deprivation alters expression of enzymes of the arachidonic and docosahexaenoic acid cascades in rat frontal cortex. Mol Psychiatry 12: 151-157.

Reshef R, Kreisel T, Beroukhim Kay D, Yirmiya R (2014). Microglia and their CX3CR1 signaling are involved in hippocampal- but not olfactory bulb-related memory and neurogenesis. Brain Behav Immun 41: 239-250.

Richard EM, Helbling JC, Tridon C, Desmedt A, Minni AM, Cador $M$ et al (2010). Plasma transcortin influences endocrine and behavioral stress responses in mice. Endocrinology 151: 649-659.

Schindelin J, Arganda-Carreras I, Frise E, Kaynig V, Longair M, Pietzsch $\mathrm{T}$ et al (2012). Fiji: an open-source platform for biological-image analysis. Nat Methods 9: 676-682.

Serhan CN, Chiang N, Dalli J, Levy BD (2014). Lipid Mediators in the Resolution of Inflammation. Cold Spring Harb Perspect Biol 7: a016311.

Su HM (2010). Mechanisms of n-3 fatty acid-mediated development and maintenance of learning memory performance. J Nutr Biochem 21: 364-373.

Weiser MJ, Wynalda K, Salem N Jr., Butt CM (2015). Dietary DHA during development affects depression-like behaviors and biomarkers that emerge after puberty in adolescent rats. J Lipid Res 56: $151-166$.

Yirmiya R, Goshen I (2011). Immune modulation of learning, memory, neural plasticity and neurogenesis. Brain Behav Immun 25: $181-213$

Zhang J, Malik A, Choi HB, Ko RW, Dissing-Olesen L, MacVicar BA (2014). Microglial CR3 activation triggers long-term synaptic depression in the hippocampus via NADPH oxidase. Neuron 82: 195-207. 